\title{
Breaking the Ice: Early Clinical Results Using Hypothermic Machine Liver Preservation
}

\author{
Tarunjeet S. Klair • James V. Guarrera
}

Published online: 15 January 2015

(C) Springer International Publishing AG 2015

\begin{abstract}
Hypothermic machine preservation (HMP) remains investigational in clinical liver transplantation. It is widely used, however, to preserve kidneys for transplantation because it has demonstrated improved results over static cold storage (CS). As with other machine preservations methods, HMP provides continuous circulation of nutrients and metabolic substrates during the ex-vivo period. Over the last few years, several groups worldwide have been actively translating HMP into the clinical arena for liver transplantation. In particular, HMP has been used for livers of varying quality, including those recovered from extended criteria donors (ECD) and donation after cardiac death (DCD) donors. Reports to date have focused on recipient hospital based HMP during the latter portion of the cold ischemic period. While some variability exists in technique, all clinical series to date have reported improved outcomes with reductions in early allograft dysfunction, biliary complications and reduced hospital length of stay. These benefits, together with the development of innovative portable HMP devices and further adoption by more centers worldwide, have "broken the ice" for more widespread use of HMP and subsequent expanded elucidation of its benefits.
\end{abstract}

This article is part of the Topical Collection on Machine Preservation of the Liver

T. S. Klair · J. V. Guarrera

Center for Liver Disease and Transplantation, Department of

Surgery, Columbia University Medical Center, New York, NY, USA

J. V. Guarrera $(\bowtie)$

Center for Liver Disease and Transplantation, Columbia University College of Physicians and Surgeons, 622 West 168th Street PH 14C, Room 202, New York, NY 10032, USA

e-mail: jjg46@cumc.columbia.edu

J. V. Guarrera

e-mail: Liverpreservation@gmail.com
Keywords Liver transplantation · Hypothermic machine preservation - Machine perfusion - Organ preservation · Extended criteria donor · Transplant outcomes

\section{Introduction}

The need for better liver allograft preservation and an expanded donor pool is being sensed with growing urgency. Not only is there an overall shortage of liver donors, but the quality of the deceased donor pool is deteriorating as the median age and incidence of comorbidities among donors increases. Eventual donors are spending more time in critical care units prior to organ recovery, resulting in greater physiologic and hemodynamic disarray. Further, organ allocation rules in some countries create additional need for the use of extended criteria donors (ECD) and donation after cardiac death donors (DCD) livers. In the USA, for instance, the likelihood of death on the waiting list is not uniform across the country. As a result, transplant centers in regions with longer waiting lists than others often rely more heavily on ECD and DCD livers [1]. Proposals to address this issue and equalize geographic disparities in organ access have the potential for creating unintended consequences, such as increased complexity of transplant logistics and "organ travel," which will increase cold ischemia times and related ramifications. The deleterious effects of transporting organs over longer distances are magnified for ECD and DCD livers. Ideally, improvements in preservation technology will negate the effects of ischemic injury, creating a more balanced physiology prior to liver transplant (LT).

Hypothermic machine perfusion (HMP) reduces the absolute insult of cold ischemia and has the potential to safely allow the lengthening of the cold ischemic time, limiting damage to donated organs and thereby improving results. Machine preservation for kidney grafts has been proven and is 
widespread in its use [2]. While liver HMP remains at an investigational stage, recent animal and human studies have demonstrated its potential utility [3]. In addition to allowing the evaluation of donated livers during the ex-vivo period, HMP could serve as a platform for ex-vivo delivery of novel therapeutic agents prior to implantation to block, or at least reduce, powerful ischemia reperfusion injury (IRI) mediators, thereby enabling further and more reliable use of marginal grafts which might otherwise be discarded.

For these reasons, the time is ripe for the consideration of expanded use of HMP and portable HMP devices, which together permit easy initiation of perfusion at the donor hospital, are safe and reliable, and alleviate the current risk to the graft associated with prolonged air and ground travel.

\section{Mechanisms of Protection}

Reperfusion of cold stored (CS) allografts and the resulting ischemia reperfusion injury (IRI) has been shown to be ameliorated by HMP. Hypoxic mitochondria and the subsequent production of reactive oxygen species (ROS) are important upstream events in transplant related IRI. ROS appear to be the basis of the IRI related inflammation instigated by the release of injurious proteins with downstream signaling and activation of endothelial, leukocyte, and Kupffer cells [4-7].

Historically, the wealth of mechanistic and molecular knowledge of liver transplant IRI processes have come from animal studies.

Recent molecular analyses of liver transplant IRI comparing HMP with CS transplanted livers from our clinical series $\left[8,9,10^{\bullet}\right]$ have added significantly to the knowledge base. Levels of expression of inflammatory cytokines, adhesion molecules, oxidative markers, and acute phase proteins were compared between HMP stored grafts and CS grafts by examining samples at the time of procurement, storage, and post-reperfusion. Decreased pro-inflammatory cytokines (TNF-a, IL-8, IL-1b) were seen in the HMP group, most likely the outcome of washout and decreased tissue hypoxia. For the HMP group, this resulted in reduced expression of adhesion markers (ICAM-1, P-selection, CXCL14, SDF-1a), CRP, and infiltration of Kupffer cells downstream. The CS group also showed more evidence of ultrastructural damage to mitochondrial and nuclear membranes. An earlier report of clinical outcomes for the same group of patients showed decreased early allograft dysfunction and other preservation related injuries [11]. These two studies $[10 \bullet, 11]$ are important as they demonstrate a direct correlation between HMP and tangible improved clinical findings, namely marked downregulation of the molecular expression of injurious mediators and cytokines that have been classically implicated in liver transplant IRI.

\section{Human Clinical Studies with HMP}

The reported clinical human experience with HMP is summarized in Table 1. Our group reported the first clinical series using HMP in a series of 20 patients comparing them to 20 controls transplanted during the same time period [11]. The grafts were low-risk in that they had good parenchymal quality and were used in patients whose lab MELD score was under 35. Vasosol solution was used as the perfusate. Vasosol is based on Belzer machine perfusion/ KPS-1 solution (Organ Recovery Systems, Itasca, IL) with additives that ameliorate IRI. The study confirmed the safety and reliability of the pump and the perfusion protocol. A representative liver undergoing HMP during the trial is shown in Fig. 1. HMP was performed in the latter half of the ex-vivo period. Peak serum AST, ALT, TBili, and SCr were all significantly lower in the HMP group and also returned to normal values more quickly. Peak recipient AST and ALT correlated to effluent enzyme levels, providing a predictive tool to assess these grafts. Early allograft dysfunction and biliary complications were significantly lower in the HMP patients who also experienced shorter hospital stays.

Reduction of IRI is most important for DCD and ECD grafts. In a recent report by the Zurich group [12••], hypothermic oxygenated perfusion (HOPE) was applied to $8 \mathrm{DCD}$ grafts. The median asystolic warm ischemia time was 18 minutes. The median perfusion time was 2 hours after a static CS time of about 2.4 hours. The portal vein was flushed with cold $\left(10{ }^{\circ} \mathrm{C}\right)$ oxygenated (40-60kPa) UW gluconate solution. The comparison group consisted of matched DBD donors. DCD recipients had lower MELD scores (6 hepatocellular carcinoma cases), 12 versus 16 , although this was not statistically significant. Outcomes were favorable and the grafts displayed excellent function, low operative blood loss, low-level transaminases, and good renal function. ICU and total hospital stays were acceptable. Most importantly, no intrahepatic biliary strictures were identified at one-year follow-up. While the study was small and this finding is surprising considering there was no active arterial perfusion, we are encouraged by the demonstrated beneficial effects of HMP on ischemic cholangiopathy in DCD liver transplantation.

Further experience from our group reported on $31 \mathrm{ECD}$ livers that were preserved with HMP [13・•]. These livers were all rejected in their originating UNOS regions or were declined by all other centers in our region. As in our pilot study, we compared HMP preserved ECD livers to matched ECD cold stored livers, but this study used a high-risk graft cohort. Average MELD scores were 19.5 (HMP) and 21.4 (CS). Cold ischemia time (CIT) was 9.3 hours versus 8.6 hours respectively. Again, dual-inflow nonpulsatile perfusion via portal 
Table 1 Clinical studies of hypothermic machine preservation in the literature

\begin{tabular}{|c|c|c|c|c|c|c|c|c|c|c|}
\hline Author & Location & Year & $\begin{array}{l}\text { Cases } \\
\text { reported } \\
\text { (n) }\end{array}$ & Donor type & Perfusion & Perfusion solution & $\begin{array}{l}\text { Oxygen } \\
\text { Delivery }\end{array}$ & $\begin{array}{l}\text { CIT } \\
(\mathrm{Hrs})\end{array}$ & $\begin{array}{l}\text { Perfusion } \\
\text { duration (Hrs) }\end{array}$ & Highlights \\
\hline $\begin{array}{c}\text { Guarrera } \\
\text { (Ref } \\
[11])\end{array}$ & $\begin{array}{l}\text { New York, } \\
\text { NY, USA }\end{array}$ & 2010 & 20 & DBD & $\begin{array}{l}\text { Arterial } \\
\text { and } \\
\text { portal }\end{array}$ & $\begin{array}{l}\text { Vasosol } \\
\text { (KPS base plus } \\
\text { IRI additives) }\end{array}$ & Ambient & 9.4 & 4.3 & $\begin{array}{l}\text { Lower EAD, } \\
\text { biliary } \\
\text { complications } \\
\text { and hospital } \\
\text { LOS in HMP }\end{array}$ \\
\hline $\begin{array}{l}\text { Dutkowski } \\
\quad \text { Ref } \\
[12 \bullet \bullet])\end{array}$ & $\begin{array}{l}\text { Zurich, } \\
\text { Switzerland }\end{array}$ & 2014 & 8 & DCD & Portal & $\begin{array}{l}\text { UW gluconate } \\
\text { (KPS) }\end{array}$ & Active & 4.6 & 2 & $\begin{array}{l}\text { No ischemic } \\
\text { cholangiopathy } \\
\text { in HMP cases }\end{array}$ \\
\hline $\begin{array}{l}\text { Guarrera } \\
\text { (Ref } \\
[13 \bullet \bullet])\end{array}$ & $\begin{array}{l}\text { New York, } \\
\text { NY, USA }\end{array}$ & 2014 & 31 & $\begin{array}{l}\text { ECD } \\
\text { orphan } \\
\text { livers }\end{array}$ & $\begin{array}{l}\text { Arterial } \\
\text { and } \\
\text { portal }\end{array}$ & $\begin{array}{l}\text { Vasosol } \\
\text { (KPS base plus } \\
\text { IRI additives) }\end{array}$ & Ambient & 9.3 & 3.8 & $\begin{array}{l}\text { Lower EAD, } \\
\text { biliary } \\
\text { complications } \\
\text { and hospital } \\
\text { LOS } \\
\text { in HMP cases }\end{array}$ \\
\hline
\end{tabular}

Abbreviations: $C I T$ cold ischemia time, $D B D$ donation after brain death, $D C D$ donation after cardiac death, $E C D$ extended criteria donor, KPS kidney perfusion solution, $E A D$ early allograft dysfunction, $L O S$ length of stay, HMP Hypothermic Machine Preservation, IRI ischemia reperfusion injury

vein and hepatic artery was used. Ambient air exchange at the organ chamber provided $\mathrm{O} 2$ with perfusate $\mathrm{PO} 2$ values over $100 \mathrm{~mm} \mathrm{Hg}$. In this series, early allograft dysfunction was reduced with HMP although this did not reach statistical significance. The rate of biliary complications in the first year was significantly lower in the HMP group ( 4 vs. $13, p=0.001$ ). Early markers of liver injury (AST, ALT, TBili) were also found to be lower in the perfused group and returned to their normal levels sooner. The incidence of acute kidney injury (per Rifle criteria), which is another indicator of morbidity post-transplant $[14,15]$, was also evaluated. HMP cases had an acute kidney injury (AKI) rate of $9.6 \%$ vs. $26 \%$ in the CS controls. There was a quicker return to normal of serum creatinine, which allowed earlier initiation of calcineurin inhibitors. In terms of potential "on-pump" viability markers, a positive correlation between effluent AST and reperfusion AST was again noted, suggesting a potential tool to pre-emptively identify grafts likely to have a more severe ischemic injury. Interestingly, two patients with the highest AST levels also had high PV pressures $(>7 \mathrm{~mm} \mathrm{Hg})$. This is another potential marker, the value of which needs to be verified with larger studies.
Fig. 1 Liver allograft undergoing perfusion during the phase 1 clinical HMP trial at Columbia University

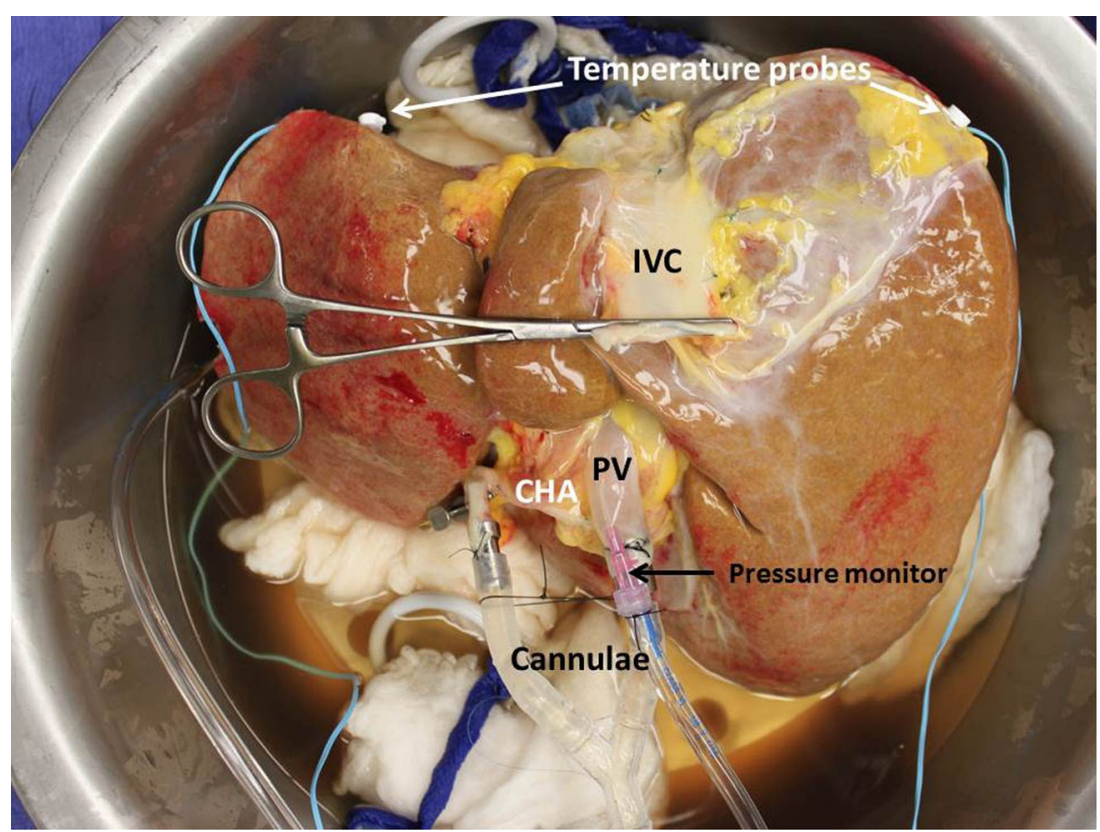




\section{What's Next?}

HMP technology has been slow to transition from the lab to the operating room, however the need for HMP is ever increasing. Out of necessity, aggressive transplant centers are using older, more steatotic, and DCD allografts with a high risk of IRI and other recipient complications. These marginal grafts are most likely to benefit from HMP.

We have finally "broken the ice" by achieving the initial goals of determining safety and feasibility of liver HMP in a clinical setting. Preliminary evidence indicates that HMP confers important protective benefits which lead to improved outcomes and may also allow safe expansion of the donor pool. Biliary complications occur in up to $30 \%$ of ECD/DCD LTs $[16,17]$, and have been long thought to be related to the quality of preservation of the biliary tree $[17,18]$. These complications represent a major source of graft failure and morbidity. In published HMP studies thus far, reduction in biliary complications have been an important finding. HMP's continuous washout of reactive oxygen species, provision of $\mathrm{O}$, and vasodilators to the peribiliary vascular plexus probably account for this protective effect in the early liver HMP experience [18]. This mechanism also accounts for benefit to DCD grafts which are the most underutilized component of the donor pool. The initial enthusiasm of DCD in liver transplantation was curbed by the difficulty in managing ischemic cholangiopathy, the occurrence of which usually requires retransplant to save the patient. Many centers remain hesitant to use these grafts and progress in DCD liver transplantation has been slow at best. The availability of HMP in addition to modified surgical techniques, such as arterial prior to portal reperfusion and t-PA infusion, holds promise in a multimodal approach that could reduce ischemic cholangiopathy rates and reinvigorate the use of DCD allografts. This pivotal goal appears achievable based on the early experience of Dutkowski and Clavien's group in Zurich.

HMP technology may also offer an opportunity to rehabilitate and use marginal grafts from remote locations where they may be more frequently rejected. In the USA, the demandsupply mismatch is skewed in certain UNOS regions where death on the waiting list is more likely. Regions with shorter waiting times are less likely to use marginal grafts. Reliable and portable novel perfusion devices are in the final stages of development. If widely deployed, these devices will allow the benefits of HMP to be brought into mainstream practice and become available to all centers.

Recently, significant interest has developed in normothermic perfusion (NMP) for liver preservation. Early experience from Peter Friend's group was presented at the World Transplant Congress in 2014 and has showed good results in 18 cases. Many hypothesize that NMP will allow significant lengthening of the ex-vivo period. While this is desirable, the portability of NMP devices and lack of backup systems (which are included in some of the HMP devices in development) make the logistics and risks of transport (especially air transport) worthy of mention. The major concern is that any technical failure of the apparatus will likely result in graft loss or contamination during the scramble to initiate CS in a nonsterile environment. While successful clinical NMP is an exciting development worthy of kudos, it appears as if the complexity, expense, logistics, and high stakes of any pump failure are issues that may limit widespread adoption especially at centers where air transport is frequently required. NMP does offer a platform for potential future sophisticated ex-vivo manipulations such as gene and reparative stem cell therapy.

HMP for renal grafts is widely used. Pump parameters are used to predict the quality of the graft and have been correlated to outcomes in some series. In both of our studies, significant correlation was found between effluent AST and postperfusion AST. Also, in our ECD series, higher portal pressures in three patients correlated with greater post perfusion enzyme leak and hemodynamic irritability upon reperfusion. In the future, this may become a viability marker which would allow triage and contribute to decisions in borderline cases where otherwise there would be no further information after the organ is removed at the donor hospital.

\section{Unanswered Questions}

Unanswered questions and untested areas in in HMP remain. In particular, each of the above studies examined the end of static cold ischemia, utilizing only a portion of the cold ischemic time available. The duration of perfusion to utilize the full potential of HMP is not known and has not been studied methodically. Additionally, the optimum level of oxygen needed in the hypothermic environment is unclear and is a point of debate between groups working on HMP in clinical and animal models. In our series, we did not use active oxygenation, but instead had an adequate $\mathrm{pO} 2$ in the perfusate (130-140 mm Hg). Furthermore, the majority of studies, including our own, have used dual perfusion which we feel is superior. Some early large animal studies also suggested that this was preferred. We believe that arterial perfusion is necessary because it is the only route to provide nutrients and substrates to the biliary tree. That being said, the Zurich group's experience with solitary portal vein perfusion did not elucidate any significant concerns. Finally, in theory, high MELD (>35), patients in the ICU should benefit the most from reduction in the physiological stresses associated with reperfusion injury. Robust early graft function would be likely to have enhanced benefits for the sickest ICU bound patients. Now that we have established the safety and benefits of HMP in low-to-high risk grafts, albeit in small numbers, the technology needs to be tested on a multicenter basis. 


\section{Conclusions}

HMP for liver has finally "broken the ice" and emerged into the clinical arena. HMP's widespread adoption will be facilitated by imminent availability of novel portable devices which allow for safe transport over long distances both on the ground and in the air. We anticipate that HMP will be an effective method for reducing the incidence of preservation injury within the most susceptible livers in the donor pool. Integrating HMP into clinical practice will likely help close the gap between organ supply and demand, and improve outcomes for liver transplantation.

\section{Compliance with Ethics Guidelines}

Conflict of Interest Tarunjeet S. Klair declares that he has no conflict of interest.

James V. Guarrera reports grants from Organ Recovery Systems. In addition, Dr. Guarrera has a patent on an Organ Perfusion Cannula issued, and a patent Organ Perfusion Device pending, and received grant support and travel/ honorarium in collaboration with Organ Recovery Systems in the development of a novel HMP system.

Human and Animal Rights and Informed Consent This article does not contain any studies with human or animal subjects performed by any of the authors.

\section{References}

Papers of particular interest, published recently, have been highlighted as:

- Of importance

-• Of major importance

1. Thuluvath PJ, Guidinger MK, Fung JJ, Johnson LB, Rayhill SC, Pelletier SJ. Liver transplantation in the United States, 1999-2008. Am J Transplant. 2010;10(4 Pt 2):1003-19 [Research Support, U.S. Gov't, P.H.S.].

2. Moers C, Smits JM, Maathuis MH, Treckmann J, van Gelder F, Napieralski BP, et al. Machine perfusion or cold storage in deceaseddonor kidney transplantation. N Engl J Med. 2009;360(1):7-19.

3. Schlegel A, Dutkowski P. Role of hypothermic machine perfusion in liver transplantation. Transplant Int. 2014; May 23. doi:10.1111/ tri.12354

4. Van Golen RF, van Gulik TM, Heger M. Mechanistic overview of reactive species-induced degradation of the endothelial glycocalyx during hepatic ischemia/reperfusion injury. Free Radic Biol Med. 2012;52:1382.

5. Zhai Y, Petrowsky H, Hong JC, Busuttil RW, Kupiec-Weglinski JW. Ischaemia-reperfusion injury in liver transplantation- from bench to bedside. Nat Rev Gastroenterol Hepatol. 2013;10:79.
6. Land WG. Emerging role of innate immunity in organ transplantation part II: potential of damage-associated molecular patterns to generate immunostimulatory dendritic cells. Transplant Rev (Orlando). 2012;26:73.

7. Schlegel A, Rougemont O, Graf R, Clavien PA, Dutkowski P. Protective mechanisms of end-ischemic cold machine perfusion in DCD liver grafts. J Hepatol. 2013;58:278-86.

8. Tulipan JE, Stone J, Samstein B, Guarrera JV, et al. Expression of acute phase mediators is attenuated by machine preservation in human liver transplantation: preliminary analysis of effluent, serum and liver biopsies. Surgery. 2011;150(2):352-60.

9. Guarrera JV, Henry SD, Chen SW, Brown T, Nachber E, Arrington $\mathrm{B}$, et al. Hypothermic machine preservation attenuates ischemia/ reperfusion markers after liver transplantation: preliminary results. J Surg Res. 2011;167(2):e365-73.

10. Henry SD, Nachber E, Tulipan J, Stone J, Bae C, Reznik L, et al. Hypothermic machine preservation reduces molecular markers of ischemia/ reperfusion injury in human liver transplantation. Am J Transplant. 2012;12(9):2477-86. This study presents a thorough evaluation of HMP's reduction of injurious ischemia reperfusion pathways in human liver transplantation.

11. Guarrera JV, Henry SD, Samstein B, Odeh-Ramadan R, Kinkhabwala M, Goldstein MJ, et al. Hypothermic machine preservation in human liver transplantation: the first clinical series. Am J Transplant. 2010;10(2):372-81 [Research Support, U.S. Gov't, P.H.S.].

12.• Dutkowski P, Schlegel A, de Oliveira M, Müllhaupt B, Neff F, Clavien PA. HOPE for human liver grafts obtained from donors after cardiac death. J Hepatol. 2014;60(4):765-72. This study describes the use of short term HMP in 8 DCD donors with excellent results.

13.• Guarrera JV, Henry SD, Samstein B, et al. Hypothermic machine preservation facilitates transplantation of "orphan" extended criteria donor livers. Am J Transplant. 2015;15:161-69. This study reports excellent results with HMP in transplanted ECD livers declined by multiple other centers.

14. O'Riordan A, Wong V, McQuillan R, McCormick PA, Hegarty JE, Watson AJ. Acute renal disease, as defined by the RIFLE criteria, post-liver transplantation. Am J Transplant. 2007;7(1):168-76 [Research Support, Non-U.S. Gov't].

15. Chen J, Singhapricha T, Hu KQ, Hong JC, Steadman RH, Busuttil $\mathrm{RW}$, et al. Postliver transplant acute renal injury and failure by the RIFLE criteria in patients with normal pretransplant serum creatinine concentrations: a matched study. Transplantation. 2011;91(3): 348-53 [Comparative Study].

16. Pine JK et al. Liver transplantation following donation after cardiac death: an analysis using matched pairs. Liver Transpl. 2009;15: 1072-82.

17. Brunner SM, Junger H, Ruemmele P, Schnitzbauer AA, Doenecke A, Kirchner GI, et al. Bile duct damage after cold storage of deceased donor livers predicts biliary complications after liver transplantation. J Hepatol. 2013;58(6):1133-9 [Research Support, NonU.S. Gov't].

18. Op den Dries S, Sutton ME, Karimian N, de Boer MT, WiersemaBuist J, Gouw AS, et al. Hypothermic oxygenated machine perfusion prevents arteriolonecrosis of the peribiliary plexus in pig livers donated after circulatory death. PLoS ONE. 2014;9(2):e88521. 